\title{
Tetramethylpyrazine ameliorates isoflurane-induced cognitive dysfunction by inhibiting neuroinflammation via miR-150 in rats
}

\author{
HUAQING CUI*, ZHONGHUI XU* and CHUNSHAN QU \\ Department of Anesthesia and Perioperative Medicine, Dongying Hospital of Traditional Chinese Medicine, \\ Dongying, Shandong 257055, P.R. China
}

Received September 11, 2019; Accepted July 10, 2020

DOI: $10.3892 / \mathrm{etm} .2020 .9110$

\begin{abstract}
Tetramethylpyrazine (TMP) has neuroprotective effects in the pathogenesis of some human diseases, such as Parkinson's disease. The present study aimed to investigate the role of TMP in isoflurane-induced cognitive dysfunction in rats, and further identify the mechanisms involved in the protective effects of TMP. The Morris water maze test was used to evaluate the cognitive function of rats exposed to isoflurane or treated with TMP. ELISA was conducted to evaluate the effects of isoflurane or TMP on neuroinflammation. The expression of microRNA-150 (miR-150) was measured using reverse transcription-quantitative PCR, and the potential target genes of miR-150 were predicted and verified. The impaired cognitive function induced by isoflurane in the rats was significantly ameliorated by treatment with TMP. In addition, TMP treatment in rats attenuated neuroinflammation caused by isoflurane. The expression of miR-150 was inhibited by isoflurane exposure, but was enhanced by TMP treatment in rats. Furthermore, the overexpression of miR-150 alleviated the isoflurane-induced cognitive dysfunction and neuroinflammation, while the neuroprotective effects of TMP were significantly abrogated by the knockdown of miR-150. AKT3 was a direct target of miR-150, and its mRNA expression was significantly decreased by the overexpression of miR-150 in isoflurane- and TMP-treated rats. These results demonstrated the protective effects of TMP against isoflurane-induced cognitive dysfunction, which were achieved by attenuating neuroinflammation via the regulation of the miR-150/AKT3 pathway. In addition, miR-150 may serve as a novel therapeutic target for the alleviation of cognitive dysfunction induced by anesthetics.
\end{abstract}

Correspondence to: Dr Chunshan Qu, Department of Anesthesia and Perioperative Medicine, Dongying Hospital of Traditional Chinese Medicine, 107 Beier Road, Dongying, Shandong 257055, P.R. China

E-mail: quchunshan198410@163.com

*Contributed equally

Key words: tetramethylpyrazine, isoflurane, cognitive dysfunction, inflammation, microRNA-150

\section{Introduction}

Anesthetic-induced cognitive dysfunction is a frequent complication following major surgery (1), and the typical symptoms include memory deficits and impaired information processing after surgery. Some patients with anesthetic-induced cognitive dysfunction can regress, but an increasing number of cases have been found to involve long-term cognitive dysfunction that leads to a significantly decreased quality of life in patients (2). For example, it is reported that $\sim 30-50 \%$ patients suffer from postoperative cognitive dysfunction after cardiac surgery, and only $45 \%$ of them can recover to baseline cognitive function by one year after surgery (3). Isoflurane is a generally used anesthetic that has been reported to induce cognitive dysfunction in patients after surgery (4). Previous studies have provided evidence for neuroinflammation as a key event in the pathogenesis of isoflurane-induced cognitive dysfunction (5). For example, increased inflammatory cytokines have been observed to be induced by anesthesia in brain after surgery (6), and isoflurane-induced cognitive dysfunction can be attenuated by suppressing neuroinflammation (7).

Tetramethylpyrazine (TMP) is an alkaloid extracted from the roots of Ligusticum chuanxiong Hort (8), and has important biological actions, including anti-inflammatory, cardioprotective, antioxidant, immune-enhanced and neuroprotective effects $(9,10)$. Moreover, TMP has been reported to exert a neuroprotective role in Parkinson's disease (11) and cerebral ischemia/reperfusion (12). However, whether TMP can also improve the cognitive dysfunction induced by isoflurane remains unknown. Previous studies have revealed that TMP can improve certain diseases by inhibiting inflammatory reactions, especially neuroinflammation. For example, Fu et al (13) found an antidepressant effect of TMP in a chronic unpredictable mild stress mouse model via the suppression of the Toll-like receptor 4 inflammatory signaling pathway. In addition, Wang et al (14) demonstrated that TMP exerts a protective role against traumatic brain injury by attenuating neuroinflammation. Thus, it was hypothesized that TMP may regulate the inflammatory reaction in isoflurane-induced cognitive dysfunction.

MicroRNAs (miRNAs/miRs) are a group of small non-coding RNAs with extensive biological function in various human diseases, such as metabolic homoeostasis (15). 
Numerous miRNAs have been identified as key molecules in neurodevelopment, such as miR-200a and miR-302c (16). In addition, certain miRNAs exhibit neuroprotective effects by regulating neuroinflammation (17). miR-150 has been suggested to be an inhibitor of neuroinflammation (18), and it can ameliorate neuropathic pain by targeting AKT3, which is a major regulator of the inflammatory response (19). However, to the best of our knowledge, the relationship between miR-150 and anesthetic-induced cognitive dysfunction has rarely been reported.

Previous studies have examined the role of miRNAs in the protective mechanisms of traditional Chinese herbal medicines in various human diseases, such as miR-294 (20). To investigate novel approaches to attenuate the impairment in cognition induced by isoflurane, the present study aimed to evaluate the protective role of TMP against isoflurane-induced cognitive dysfunction in rats, and to further identify the underlying mechanism by assessing the role of miR-150 and its effects on neuroinflammation.

\section{Materials and methods}

Animals and exposure to isoflurane. In total, 100 male Sprague-Dawley at 7 weeks of age (220-260 g) rats were purchased from the Animal Center of Chinese Academy of Sciences, and were housed in animal facilities with free access to water and food under a standard environment $\left(25 \pm 1^{\circ} \mathrm{C}\right.$, $60-65 \%$ humidity and 12/12 h light/dark cycle). The rats were randomly designed into two groups: Control $(n=10)$ and isoflurane groups $(n=90)$. For isoflurane exposure, the rats were housed in anesthetic chambers containing $2 \%$ isoflurane (Sigma-Aldrich; Merck KGaA) mixed with $100 \%$ oxygen for $4 \mathrm{~h}$. The rats in the control group were placed in the animal facilities with room air. All the animal experiments were in accordance with the guidelines of the National Institutes of Health and Association for Assessment and Accreditation of Laboratory Animal Care (21), and were approved by the Animal Care and Use Committee of Dongying Hospital of Traditional Chinese Medicine Hospital.

Animal treatment. The rats that received isoflurane exposure were further assigned to eight groups (10 rats/group): Isoflurane + DMSO vehicle, isoflurane + TMP, isoflurane + miR-negative control (miR-NC), isoflurane + miR-150 mimic, isoflurane + miR-150 inhibitor, isoflurane + TMP + miR-NC, isoflurane + $\mathrm{TMP}+\mathrm{miR}-150$ inhibitor and isoflurane groups. For DMSO or TMP treatment, the rats were intraperitoneally injected with DMSO $(1 \mathrm{ml})$ or $20 \mathrm{mg} / \mathrm{kg}$ TMP (Nanjing Zelang Medical Technology Co., Ltd.) at $24 \mathrm{~h}$ after the isoflurane treatment (22). The miR-150 mimic, miR-150 inhibitor and miR-NC (2 nM) were synthesized by Shanghai GenePharma Co., Ltd. and injected into the rats by lateral cerebroventricular injection to regulate the expression of miR-150 in vivo $24 \mathrm{~h}$ before isoflurane exposure, Lipofectamine 2000 (Thermo Fisher Scientific, Inc.) was used for transfection according to the manufacturer's instructions.. The sequences for the vectors were as follows: miR-150 mimic, 5'-UCUCCCAACCCUUGUACCAGUG-3'; miR-150 inhibitor, 5'-CACUGGUACAAGGGUUGGGAG A-3'; and miR-NC, 5'-UUCUCCGAACGUGUCACGU-3'. The detailed treatment for the groups is summarized in Table I.
After treatment, five rats from each group were subjected to the Morris water maze test to evaluate the cognitive function, and hippocampi were collected from the remaining five rats after sacrifice by cervical dislocation.

Morris water maze performance. This study evaluated the cognitive function of the rats using a Morris water maze test, which was performed using a method previously described (23). Briefly, $48 \mathrm{~h}$ post-isoflurane exposure, the rats in each group were subjected to the hidden-platform training for 5 days with 2 trials per day, and the platform was removed on the 6th day. A computerized tracking system (Actimetrics software version 2.6; Actimetrics) was used to monitor the trials, and the performance, including latency to find the hidden platform, distance traveled, time spent in each quadrant and number of platform crossing, of the rats in different groups was recorded and compared. After the test, the rats (weight, 250-300 g) were sacrificed by cervical dislocation under anesthesia with $50 \mathrm{mg} / \mathrm{kg}$ pentobarbitone.

ELISA. The expression levels of the pro-inflammatory cytokines IL-1 $\beta$ (cat. no. RLB00), IL-6 (cat. no. R6000B) and TNF- $\alpha$ (cat. no. RTA00) in the hippocampus collected from the rats in each group were detected using the rat ELISA kit (Invitrogen; Thermo Fisher Scientific, Inc.), following the manufacturer's instruction.

$R N A$ extraction and reverse transcription-quantitative $P C R$ $(R T-q P C R)$. Total RNAs in the hippocampus were extracted using TRIzol ${ }^{\circledR}$ reagent (Invitrogen; Thermo Fisher Scientific, Inc.), which were then used for RT to synthesize cDNA using the PrimeScript RT reagent kit (Takara Bio, Inc.) according to the manufacturer's protocols, the conditions were $37^{\circ} \mathrm{C}$ for $15 \mathrm{~min}$ and $98^{\circ} \mathrm{C}$ for $5 \mathrm{~min}$. Hippocampal expression levels of miR-150 and the mRNA expression of AKT3 were measured using RT-qPCR, which was performed using a SYBR green I Master Mix kit (Invitrogen; Thermo Fisher Scientific, Inc.) on a 7500 Real-Time PCR system (Applied Biosystems; Thermo Fisher Scientific, Inc.). The PCR conditions were as follows: $95^{\circ} \mathrm{C}$ for $5 \mathrm{~min}$, followed by 40 cycles of $95^{\circ} \mathrm{C}$ for $30 \mathrm{sec}, 60^{\circ} \mathrm{C}$ for $30 \mathrm{sec}$, and $72^{\circ} \mathrm{C}$ for $40 \mathrm{sec}$ and then a final extension at $72^{\circ} \mathrm{C}$ for $5 \mathrm{~min}$. The final expression levels were calculated using the $2^{-\Delta \Delta \mathrm{Cq}}$ method (24) and normalized to U6 or GAPDH. Primers used in the current study are as follows: miR-150, forward: 5'-TGCGGTCTCCCAACCCTTG-3' and reverse, 5'-CCAGTG CAGGGTCCGAGGT-3'; AKT3, forward, 5'-TGTGGATTT ACCTTATCCCCTCA-3' and reverse, 5'-GTTTGGCTTTGG TCGTTCTGT-3'; U6, forward, 5'-CGCTTCGGCAGCACA TATACTAAAATTGGAAC-3' and reverse: 5'-GCTTCACGA ATTTGCGTGTCATCCTTGC-3'; GAPDH, forward, 5'-AGA AGGCTGGGGCTCATTTG-3' and reverse, 5'-AGGGGC CATCCACAGTCTTC-3'.

Cell culture. Human neuroblastoma SH-SY5Y cells (cat. no. ATCC ${ }^{\circledR}$ CRL-2266 ${ }^{\mathrm{TM}}$ ) were purchased from the American Type Culture Collection. Cells were cultured in DMEM (Gibco; Thermo Fisher Scientific, Inc.) containing 10\% FBS (Gibco; Thermo Fisher Scientific, Inc.) and 1\% penicillin/streptomycin (Gibco; Thermo Fisher Scientific, Inc.) at $37^{\circ} \mathrm{C}$ in a humidified incubator with $5 \% \mathrm{CO}_{2}$. 
Table I. Treatment in different groups.

Groups

Treatment

Control $(n=10)$
Isoflurane $(n=10)$
Isoflurane + DMSO $(n=10)$
Isoflurane + TMP $(n=10)$
Isoflurane + miR-NC $(n=10)$
Isoflurane + miR-150 mimic $(n=10)$
Isoflurane + miR-150 inhibitor $(n=10)$
Isoflurane + TMP + miR-NC $(n=10)$
Isoflurane + TMP + miR-150 mimic $(n=10)$
Isoflurane + TMP + miR-150 inhibitor $(n=10)$

Room air
$2 \%$ isoflurane exposure
$2 \%$ isoflurane exposure and DMSO injection
$2 \%$ isoflurane exposure and injection with $20 \mathrm{mg} / \mathrm{kg}$ TMP
$2 \%$ isoflurane exposure and injection with $2 \mathrm{nM} \mathrm{miR}-\mathrm{NC}$
$2 \%$ isoflurane exposure and injection with $2 \mathrm{nM} \mathrm{miR}-150$ mimic
$2 \%$ isoflurane exposure and injection with $2 \mathrm{nM} \mathrm{miR}-150$ inhibitor
$2 \%$ isoflurane exposure and injection with $20 \mathrm{mg} / \mathrm{kg}$ TMP and $2 \mathrm{nM}$ miR-NC
$2 \%$ isoflurane exposure and injection with $20 \mathrm{mg} / \mathrm{kg}$ TMP and $2 \mathrm{nM}$ miR-150
mimic
$2 \%$ isoflurane exposure and injection with $20 \mathrm{mg} / \mathrm{kg}$ TMP and $2 \mathrm{nM} \mathrm{miR-150}$
inhibitor

TMP, tetramethylpyrazine; miR, microRNA; NC, negative control.

Dual-luciferase reporter assay. The potential targets of miR-150 were predicted using TargetScan 7.2 (http://www. targetscan.org/vert_72/), which are presented in Table II, and the 3'-untranslated region (UTR) of AKT3 had the complementary sequence of miR-150. To assess their interaction, a dual-luciferase reporter assay was conducted. The wild-type (WT) and mutant type (MUT) 3'-UTR of AKT3 were cloned into pGL3 reporter vector (50 ng; Promega Corporation) (Sangon Biotech Co., Ltd.) and then were respectively co-transfected into SH-SY5Y cells with miR-150 mimic, miR-150 inhibitor or miR-NC (200 ng) using Lipofectamine ${ }^{\circledR} 2000$ (Invitrogen; Thermo Fisher Scientific, Inc.), following the manufacturer's instruction. After cell transfection for $48 \mathrm{~h}$, the relative luciferase activity was measured using the Promega Dual-luciferase Reporter Assay kit (Promega Corporation). Renilla luciferase activity was used as the internal control.

Statistical analysis. Data are presented as the mean \pm SD, and were analyzed using SPSS 21.0 (IBM Corp.) and GraphPad Prism 7.0 software (GraphPad Software, Inc.). The differences between groups were assessed using Student's t-test or one-way ANOVA, followed by Tukey's or Bonferroni post hoc test. Moreover, behavioral data were analyzed using a mixed ANOVA and a Bonferroni post hoc test. $\mathrm{P}<0.05$ was considered to indicate a statistically significant difference. Each experiment was repeated at least three times.

\section{Results}

Neuroprotective effects of TMP on isoflurane-induced cognitive dysfunction. A Morris water maze test was performed to evaluate the effects of isoflurane and TMP treatment on the cognitive function in the rats. In rats exposed to isoflurane, the latency to find the hidden platform and the distance travelled during the test were significantly increased compared with the control group (all $\mathrm{P}<0.05$; Fig. 1A and $\mathrm{B}$ ), indicating an impairment in the spatial learning ability of the rats. After removing the hidden platform, it was found that the rats exposed to isoflurane spent less time in target quadrant and crossed through the original platform location fewer times compared with the control group (all $\mathrm{P}<0.05$; Fig. $1 \mathrm{C}$ and $\mathrm{D}$ ), suggesting that the memory ability of the rats was impaired. Moreover, the injection of TMP significantly rescued the isoflurane-induced cognitive dysfunction, as demonstrated by the improved spatial learning and memory indicators in the Morris water maze test (all $\mathrm{P}<0.05$; Fig. 1).

Anti-inflammatory effect of TMP in rats exposed to isoflurane. The impairment in cognitive function induced by isoflurane is accompanied by enhanced inflammatory responses (1). The hippocampal levels of the pro-inflammatory cytokines IL-1 $\beta$, IL- 6 and TNF- $\alpha$ were all increased by exposure to isoflurane (all $\mathrm{P}<0.05$; Fig. 2). However, in the rats that received TMP treatment, the isoflurane-induced increases in IL-1 $\beta$, IL- 6 and TNF- $\alpha$ levels were significantly reversed (all $\mathrm{P}<0.05$; Fig. 2).

Dysregulation of miR-150 in rats under isoflurane anesthesia and TMP treatment. The aberrant expression of miR-150 has been identified in neuropathic pain (19). The results demonstrated that the expression of miR-150 in the hippocampus was significantly decreased in the rats exposed to isoflurane compared with control group ( $\mathrm{P}<0.05$; Fig. 3 ), while this effect was partially abrogated by TMP treatment $(\mathrm{P}<0.05$; Fig. 3).

Effects of miR-150 on isoflurane-induced cognitive dysfunction and neuroinflammation. Based on the decreased expression of miR-150 in rats exposed to isoflurane, the effects of miR-150 on the impaired cognitive function and neuroinflammation induced by isoflurane were further investigated. The expression of miR-150 was upregulated by the miR-150 mimic but was downregulated by the miR-150 inhibitor in hippocampal tissues in vivo (both $\mathrm{P}<0.05$; Fig. $4 \mathrm{~A}$ ). It was found that isoflurane-induced spatial learning and memory impairments were all reversed by the overexpression of miR-150, but were aggravated by the knockdown of miR-150 (all $\mathrm{P}<0.05$; Fig. 4B-E). Moreover, the hippocampal concentrations of IL-1 $\beta$, IL- 6 and 
Table II. List of the predicted target genes of miR-150 commonly predicted in TargetScan 7.2 database.

\begin{tabular}{|c|c|c|}
\hline & & Predicted target genes of miR-150 \\
\hline & Target gene & Gene name \\
\hline 1 & MYB & v-myb avian myeloblastosis viral oncogene homolog \\
\hline 2 & ADIPOR2 & Adiponectin receptor 2 \\
\hline 3 & HILPDA & Hypoxia inducible lipid droplet-associated \\
\hline 4 & TADA1 & Transcriptional adaptor 1 \\
\hline 5 & MTCH2 & Mitochondrial carrier 2 \\
\hline 6 & MBD6 & methyl-CpG binding domain protein 6 \\
\hline 7 & BASP1 & Brain abundant, membrane attached signal protein 1 \\
\hline 8 & ZBTB4 & Zinc finger and BTB domain containing 4 \\
\hline 9 & ZMAT2 & Zinc finger, matrin-type 2 \\
\hline 10 & PLP2 & Proteolipid protein 2 (colonic epithelium-enriched) \\
\hline 11 & FOXD3 & Forkhead box D3 \\
\hline 12 & ZFP91 & ZFP91 zinc finger protein \\
\hline 13 & NKX2-4 & NK2 homeobox 4 \\
\hline 14 & PTP4A1 & Protein tyrosine phosphatase type IVA, member 1 \\
\hline 15 & KCNIP1 & Kv channel interacting protein 1 \\
\hline 16 & SGMS1 & Sphingomyelin synthase 1 \\
\hline 17 & EIF4B & Eukaryotic translation initiation factor 4B \\
\hline 18 & ETF1 & Eukaryotic translation termination factor 1 \\
\hline 19 & PRICKLE2 & Prickle homolog 2 (Drosophila) \\
\hline 20 & STX5 & Syntaxin 5 \\
\hline 21 & ZEB1 & Zinc finger E-box binding homeobox 1 \\
\hline 22 & ACVR1B & Activin A receptor, type IB \\
\hline 23 & ARIH2 & Ariadne RBR E3 ubiquitin protein ligase 2 \\
\hline 24 & AKT3 & v-akt murine thymoma viral oncogene homolog 3 \\
\hline 25 & PDIA6 & Protein disulfide isomerase family A, member 6 \\
\hline 26 & GABRA4 & $\gamma$-aminobutyric acid A receptor, $\alpha 4$ \\
\hline 27 & CDAN1 & Codanin 1 \\
\hline 28 & FOXO4 & Forkhead box $\mathrm{O} 4$ \\
\hline 29 & UBE2R2 & Ubiquitin-conjugating enzyme E2R 2 \\
\hline 30 & DYRK1A & Dual-specificity tyrosine-(Y)-phosphorylation regulated kinase 1A \\
\hline 31 & HMGA2 & High mobility group AT-hook 2 \\
\hline 32 & MBTD1 & mbt domain containing 1 \\
\hline 33 & EP300 & E1A binding protein p300 \\
\hline 34 & RORB & RAR-related orphan receptor B \\
\hline 35 & GOSR1 & Golgi SNAP receptor complex member 1 \\
\hline 36 & NEFM & Neurofilament, medium polypeptide \\
\hline 37 & FOXP1 & forkhead box P1 \\
\hline 38 & SRGAP3 & SLIT-ROBO Rho GTPase activating protein 3 \\
\hline 39 & $\mathrm{HIF}-1 \alpha$ & Hypoxia inducible factor- $1 \alpha$ subunit (basic helix-loop-helix transcription factor) \\
\hline 40 & LMO4 & LIM domain only 4 \\
\hline 41 & EPHB2 & $\mathrm{EPH}$ receptor $\mathrm{B} 2$ \\
\hline 42 & NEGR1 & Neuronal growth regulator 1 \\
\hline 43 & UST & Uronyl-2-sulfotransferase \\
\hline 44 & ATP8A2 & ATPase, aminophospholipid transporter, class I, type 8A, member 2 \\
\hline 45 & TMCC1 & Transmembrane and coiled-coil domain family 1 \\
\hline 46 & RAD23B & RAD23 homolog B (S. cerevisiae) \\
\hline 47 & PAPD5 & PAP associated domain containing 5 \\
\hline 48 & CPD & Carboxypeptidase D \\
\hline 49 & EBF3 & Early B-cell factor 3 \\
\hline 50 & SMARCD1 & SWI/SNF related, matrix associated, actin dependent regulator of chromatin, subfamily d, member 1 \\
\hline
\end{tabular}


Table II. Continued.

Predicted target genes of miR-150

\begin{tabular}{lll}
\cline { 2 - 3 } & Target gene & \multicolumn{1}{c}{ Gene name } \\
\hline 51 & ITSN1 & Intersectin 1 (SH3 domain protein) \\
52 & GDI1 & GDP dissociation inhibitor 1 \\
53 & FAM134C & Family with sequence similarity 134, member C \\
54 & TBC1D20 & TBC1 domain family, member 20 \\
55 & MMP14 & Matrix metallopeptidase 14 (membrane-inserted) \\
56 & GABRG2 & $\gamma$-aminobutyric acid A receptor, $\gamma 2$ \\
57 & ADAM19 & ADAM metallopeptidase domain 19 \\
58 & EIF4E & Eukaryotic translation initiation factor 4E \\
59 & TET3 & tet methylcytosine dioxygenase 3 \\
60 & PKHD1 & Polycystic kidney and hepatic disease 1 (autosomal recessive) \\
61 & PAPPA & Pregnancy-associated plasma protein A, pappalysin 1 \\
62 & CHD2 & Chromodomain helicase DNA binding protein 2 \\
63 & CC2D1B & Coiled-coil and C2 domain containing 1B \\
64 & PRRT2 & Proline-rich transmembrane protein 2 \\
65 & MMP16 & Matrix metallopeptidase 16 (membrane-inserted) \\
\hline
\end{tabular}

A

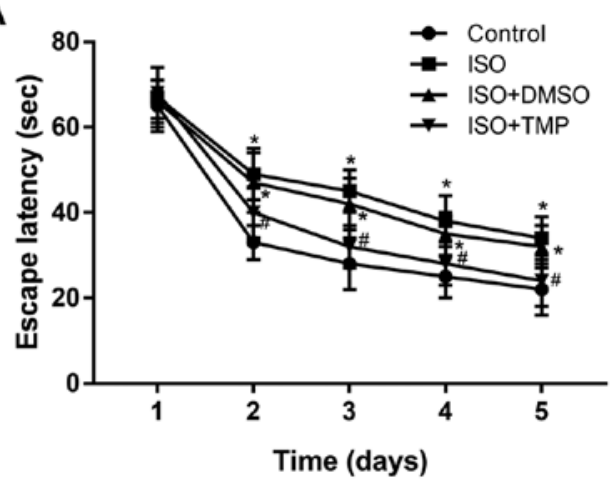

C

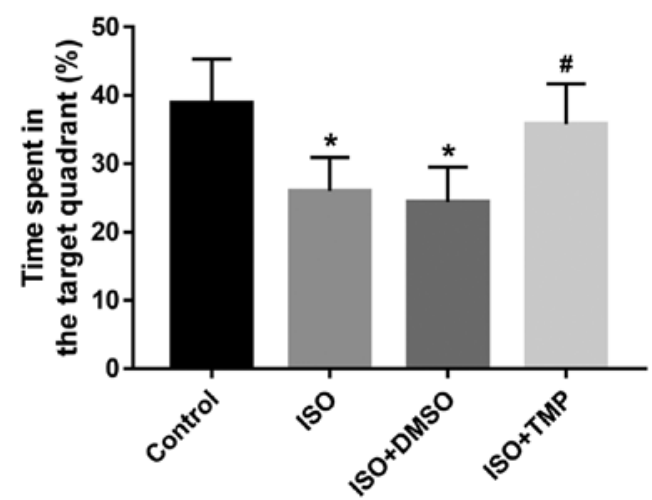

B

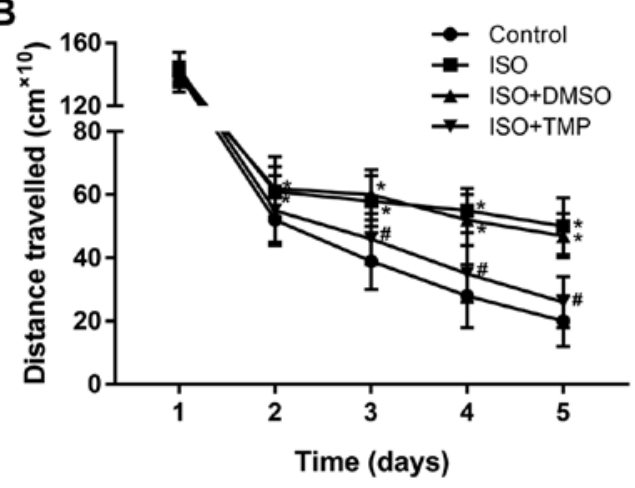

D

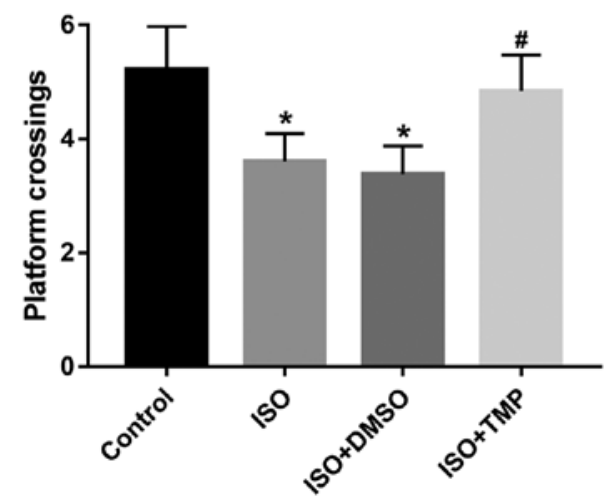

Figure 1. TMP treatment ameliorates ISO-induced cognitive dysfunction in rats. (A) Latency to reach the platform and (B) the distance travelled by the rats were measured to evaluate the spatial learning ability of the rats under ISO exposure or TMP treatment. (C) Time spent in the target quadrant and (D) number of crossing of the original platform were analyzed to evaluate the spatial memory of the rats exposed to ISO or treated with TMP. "P<0.05 vs. Control; ${ }^{\text {"}} \mathrm{P}<0.05$ vs. ISO. ISO, isoflurane; TMP, tetramethylpyrazine.

TNF- $\alpha$ were ameliorated by the upregulation of miR-150, but the opposite results were obtained in the rats with miR-150 knockdown (all $\mathrm{P}<0.05$; Fig. 4F).
TMP exerts neuroprotective effects by regulating $\mathrm{miR}-150$ in rats exposed to isoflurane. Considering the regulatory activity of TMP on the expression of miR-150 in the isoflurane-treated 

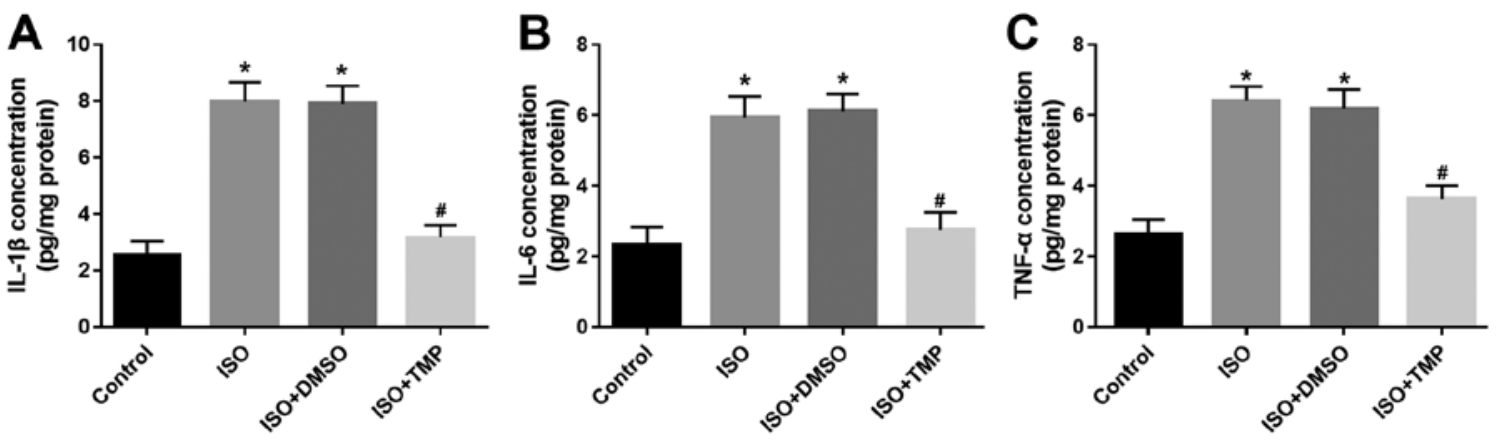

Figure 2. TMP treatment attenuates neuroinflammation induced by ISO exposure in rats. Hippocampal levels of (A) IL-1 $\beta$, (B) IL-6 and (C) TNF- $\alpha$ were measured to determine the inflammatory response in rats. ${ }^{*} \mathrm{P}<0.05$ vs. Control; ${ }^{*} \mathrm{P}<0.05 \mathrm{vs}$. ISO. ISO, isoflurane; TMP, tetramethylpyrazine.

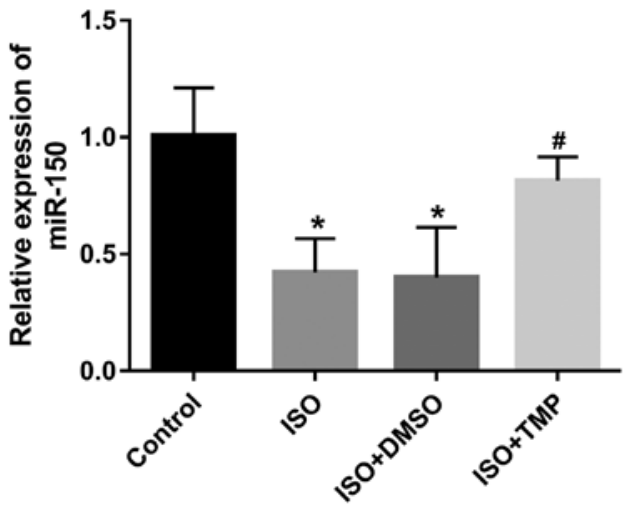

Figure 3. Effects of ISO and TMP on the expression of miR-150 in rats. The expression of miR-150 in the hippocampus was significantly decreased in the rats exposed to isoflurane compared with control group, while this effect was partially abrogated by TMP treatment. " $\mathrm{P}<0.05$ vs. Control; ${ }^{\mathrm{P}}<0.05$ vs. ISO. ISO, isoflurane; TMP, tetramethylpyrazine.

rats, the role of miR-150 in the neuroprotective effect of TMP in rats was evaluated. In the rats exposed to isoflurane, the increased expression of miR-150 induced by TMP was successfully inhibited by the miR-150 inhibitor $(\mathrm{P}<0.05$; Fig. 5A). Furthermore, the results obtained from the Morris water maze test indicated that the neuroprotective effects of TMP on the cognitive function in the rats exposed to isoflurane were significantly attenuated by the knockdown of miR-150 (all $\mathrm{P}<0.05$; Fig. 5B-E). With regards to neuroinflammation, the decreased hippocampal levels of IL-1 $\beta$, IL- 6 and TNF- $\alpha$ induced by TMP were all upregulated by the miR-150 inhibitor (all $\mathrm{P}<0.05$; Fig. 5F).

AKT3 serves as a target gene of miR-150. miR-150 mimic transfection significantly increased the expression of miR-150 in SH-SY5Y cells, but miR-150 inhibitor transfection had an opposite effect (all P $<0.05$; Fig. 6A). A complementary sequence of miR-150 was found in the 3'-UTR of AKT3 (Fig. 6B). The luciferase analysis results demonstrated that the overexpression of miR-150 could inhibit the relative luciferase activity, but the knockdown of miR-150 could promote the luciferase activity in the WT group (all P<0.05; Fig. 6C). However, no significant changes were identified in the luciferase activity in MUT groups (all $\mathrm{P}>0.05$ ).

The relative mRNA expression of AKT3 in the hippocampus collected from the experimental rats was measured.
It was demonstrated that isoflurane exposure led to increased expression of AKT3, while TMP treatment could inhibit this increase in AKT3 expression (all $\mathrm{P}<0.05$; Fig. 6D). Furthermore, via the in vivo regulation of miR-150, the mRNA expression of AKT3 in the isoflurane-treated rats was inhibited by the overexpression of miR-150, but was enhanced by the knockdown of miR-150 compared with the isoflurane group (all $\mathrm{P}<0.05$; Fig. 6D). In the isoflurane-treated rats that received both TMP and miR-150 inhibitor administration, the TMP-induced decrease in AKT3 expression was reversed by the knockdown of miR-150 (P<0.05; Fig. 6D).

\section{Discussion}

Anesthetic-induced cognitive dysfunction remains a challenging health problem for doctors after major surgery (25). The present study focused on the protective effects of the traditional Chinese herbal medicine TMP in rats exposed to isoflurane and evaluated the underlying mechanisms. The current results suggested that the TMP treatment in rats ameliorated the impairment in cognitive function induced by isoflurane exposure, and that anesthetic-induced neuroinflammation was also attenuated by TMP. In addition, it was found that the hippocampal expression of miR-150 was suppressed by isoflurane exposure, but was enhanced after TMP treatment. The overexpression of miR-150 in rats led to improved cognitive function and neuroinflammation, while the knockdown of miR-150 abrogated the protective effects of TMP against isoflurane-induced cognitive dysfunction and neuroinflammation.

Previous studies have revealed the clinical potential of traditional Chinese herbal medicines in various human diseases, such as hepatitis and neurodegenerative disease (26). Some active components of Chinese medicine can exert significantly protective effects for the treatment of cognitive function impairment induced by anesthetics. For example, Chen et al (1) provided evidence for apigenin as a medicine that attenuates cognitive dysfunction induced by isoflurane in aged rats. Moreover, Zhang et al (27) demonstrated the neuroprotective role of ginsenoside $\mathrm{Rg} 1$ in rats exposed to isoflurane, while Chen et al (28) revealed that vitexin exerts a protective effect against neurotoxicity induced by isoflurane. The neuroprotective role of salidroside has also been reported in rats expose to isoflurane (29). These aforementioned studies provide evidence for the important roles of traditional Chinese medicine in neuroprotective effects in rats exposed to isoflurane. 

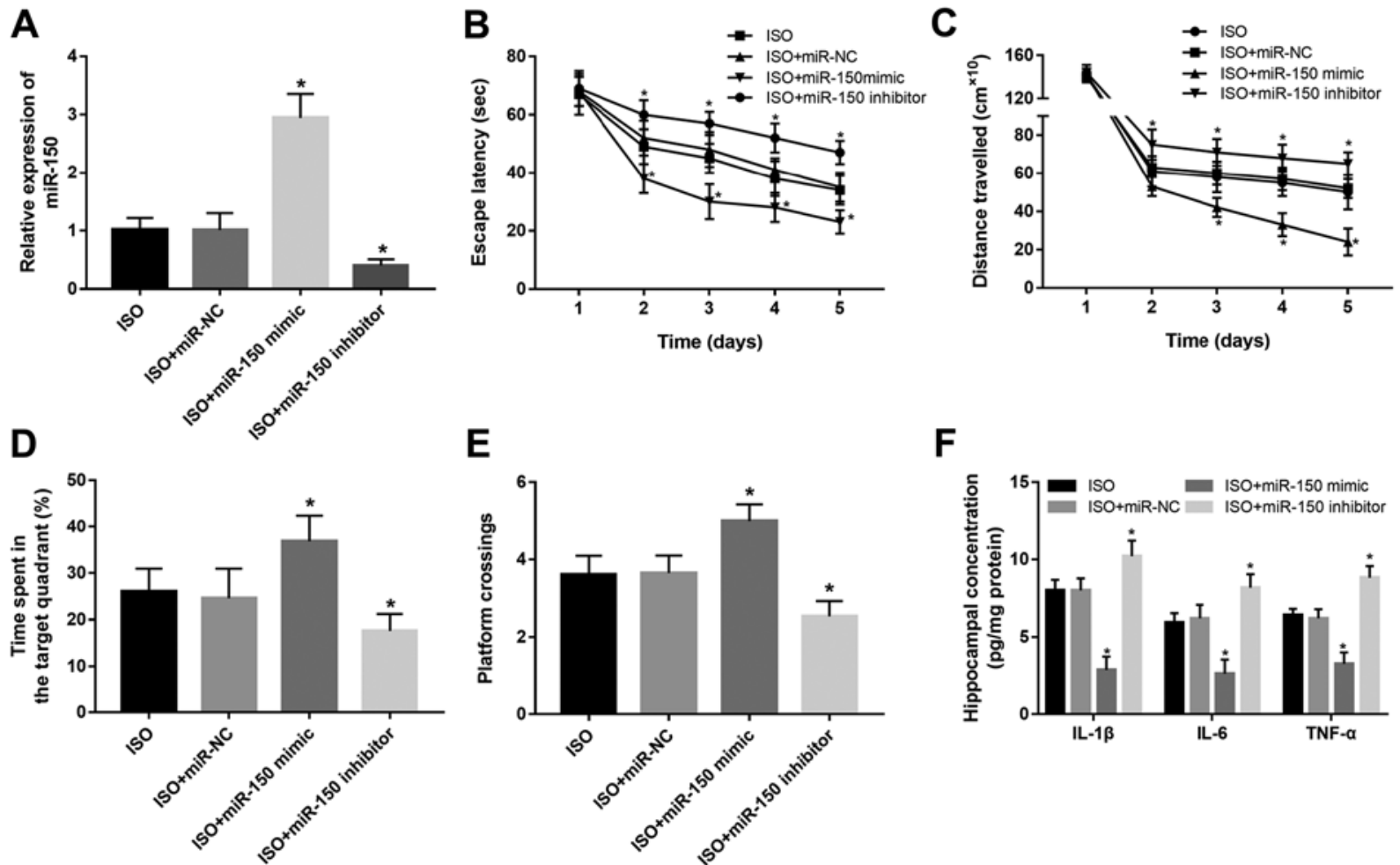

$\mathbf{F}$

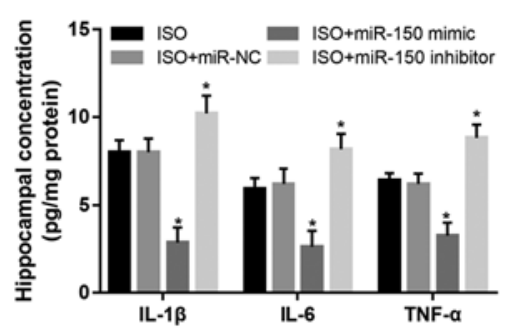

Figure 4. Effects of miR-150 on cognitive dysfunction and neuroinflammation in rats exposed to ISO. (A) Efficiency of in vivo transduction of the miR-150 mimic and miR-150 inhibitor. Regulatory effects of miR-150 on the (B) escape latency, (C) distance travelled, (D) time spent in the target quadrant and (E) the number of crossings of the original platform in the rats that received ISO exposure. (F) Hippocampal concentrations of IL-1 $\beta$, IL- 6 and TNF- $\alpha$ were regulated by miR-150 in rats exposed to ISO. ${ }^{*} \mathrm{P}<0.05$ vs. ISO. ISO, isoflurane; NC, negative control; miR, microRNA.
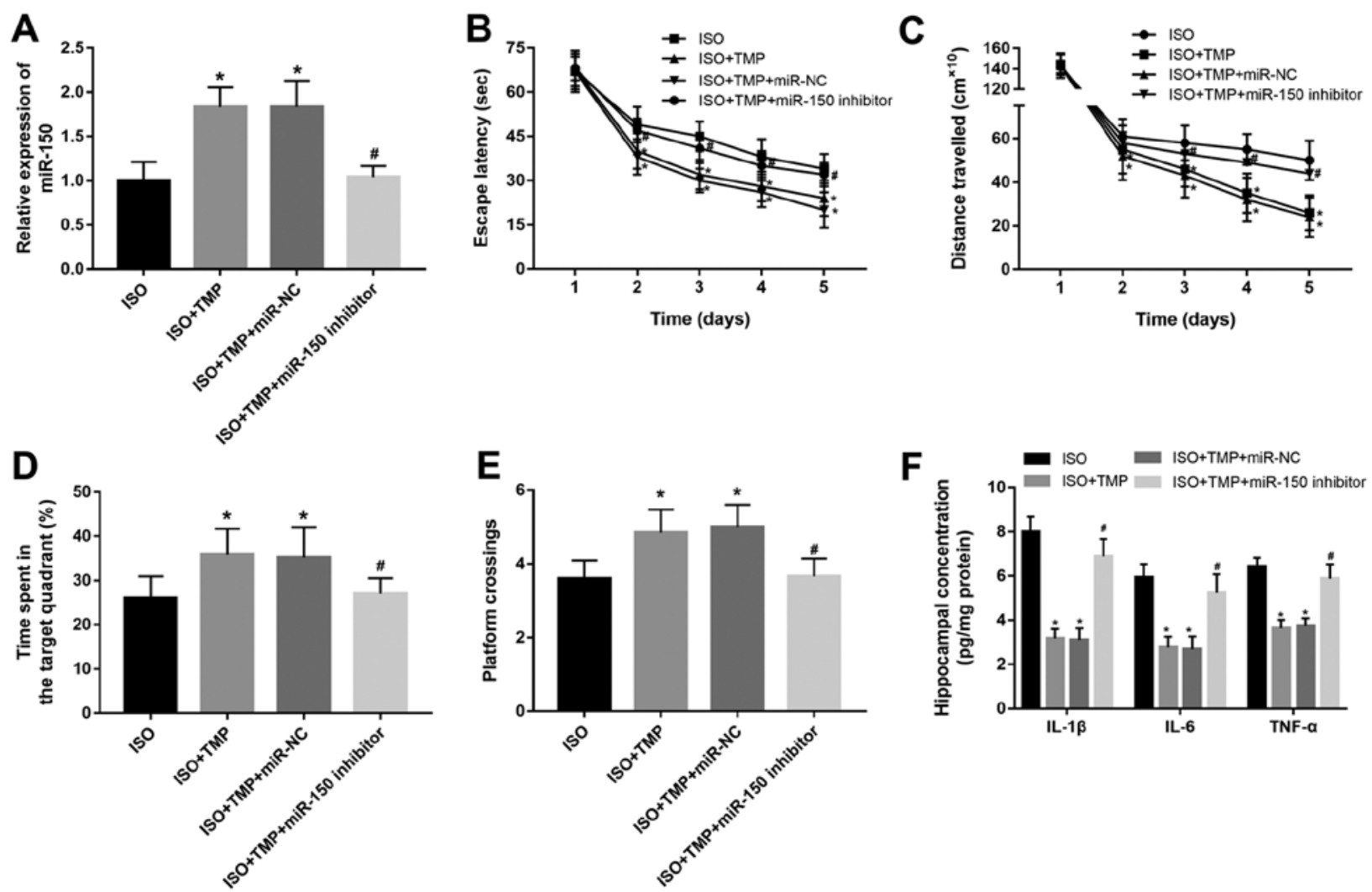

Figure 5. miR-150 mediates the protective effects of TMP on the cognitive dysfunction and neuroinflammation induced by ISO in rats. (A) Upregulated expression of miR-150 induced by TMP was decreased by miR-150 inhibitor in ISO-treated rats. Effects of TMP treatment on the (B) escape latency, (C) distance travelled, (D) time spent in the target quadrant and (E) platform crossings were all abrogated by miR-150 knockdown in rats. (F) Ameliorative effects of TMP on ISO-induced neuroinflammation were reversed by miR-150 knockdown. ${ }^{*} \mathrm{P}<0.05$ vs. ISO; ${ }^{\#} \mathrm{P}<0.05$ vs. ISO + TMP. ISO, isoflurane; TMP, tetramethylpyrazine; NC, negative control; miR, microRNA. 

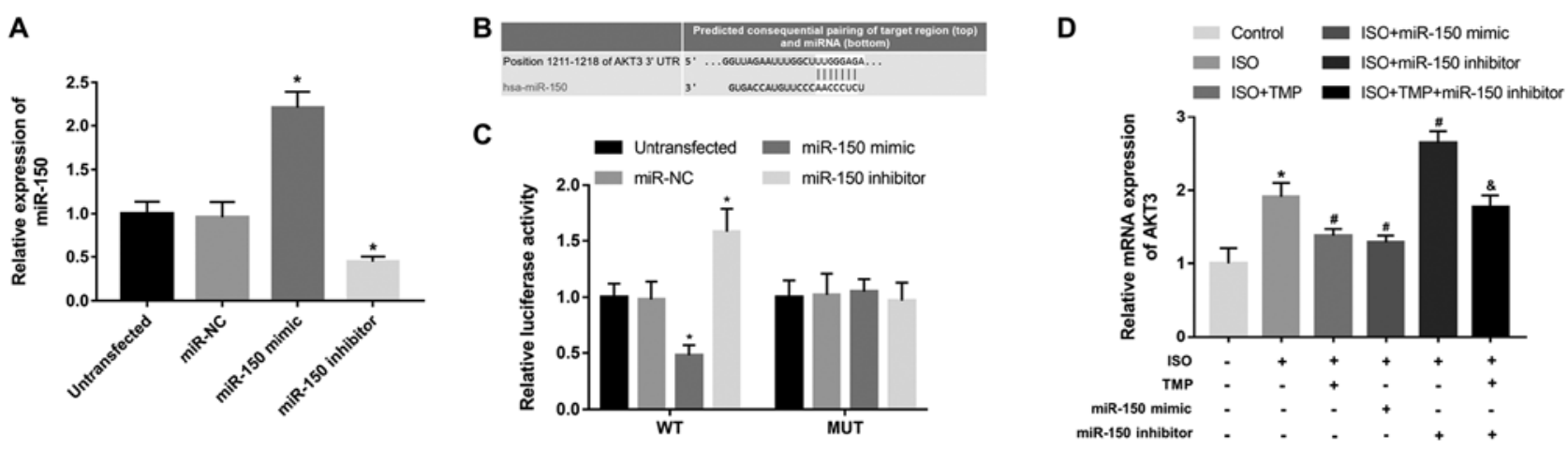

Figure 6. miR-150 directly regulates the expression of AKT3. (A) miR-150 mimic transfection significantly increased the expression of miR-150 in SH-SY5Y cells, but miR-150 inhibitor transfection has an opposite effect. (B) Complementary sequence of miR-150 in the 3'-UTR of AKT3. (C) Luciferase activity analysis to assess the interaction between miR-150 and AKT3. ${ }^{*} \mathrm{P}<0.05$ vs. untransfected group. (D) ISO exposure led to increased AKT3 expression, while this inhibition was rescued by TMP treatment. The overexpression of miR-150 could inhibited, whereas the knockdown of miR-150 could enhanced the expression of AKT3 The TMP-induced inhibition in AKT3 expression was reversed by the knockdown of miR-150. ${ }^{*} \mathrm{P}<0.05$ vs. Control; ${ }^{*} \mathrm{P}<0.05$ vs. ISO; ${ }^{\circledR} \mathrm{P}<0.05$ vs. ISO + TMP. ISO, isoflurane; TMP, tetramethylpyrazine; UTR, untranslated region; NC, negative control; miR, microRNA; WT, wild-type; MUT, mutant.

TMP is a widely used and analyzed traditional Chinese medicine in human diseases, such as liver fibrosis (30) and prostate cancer (31). The effects of TMP on the nervous system have been reported in oxygen-glucose deprivation-induced mortality (8) and ischemic stroke (32). However, whether TMP is involved in the neuroregulation in rats exposed to isoflurane remains elusive. In the current study, a Morris water maze test was performed to evaluate the effect of TMP on the spatial learning and memory abilities of rats. It was demonstrated that the impaired cognitive function induced by isoflurane in rats was significantly ameliorated by TMP treatment. Thus, it was concluded that TMP exerts a protective effect against isoflurane-induced cognitive dysfunction.

Neuroinflammation is a key event during impairments of learning and memory in rats exposed to anesthetics (33). The neuroprotective effects of apigenin (1) and ginsenoside $\mathrm{Rb1}$ (34) in rats exposed to isoflurane are achieved by attenuating neuroinflammation. TMP has been shown to be closely associated with inflammation, and its anti-inflammatory effect has been well described in vivo (35). The present results also provide evidence that TMP exerts an anti-neuroinflammatory role in rats exposed to isoflurane, and suggested that TMP may ameliorate isoflurane-induced cognitive dysfunction by attenuating neuroinflammation. However, the changes in systemic inflammatory responses were not analyzed, which was one of the limitations of the current study. Despite the presence of a blood-brain barrier, it has been reported that the circulating immune system is also closely associated with neuroinflammation (36). Thus, further studies should investigate the effects of TMP on systemic inflammatory responses to further identify the regulatory mechanisms.

Previous studies have investigated the role of miRNAs in the mechanisms involved in the protective effects of traditional Chinese medicine. For instance, Liu et al (37) revealed that curcumin can enhance sensitivity to radiation in prostate cancer cells via the regulation of miR-143. Moreover, Fan et al (38) demonstrated that emodin protects against hyperglycemia-induced injury in PC-12 cells via the upregulation of miR-9. TMP has been reported to alleviate spinal cord injury via the regulation of miR-214-3p (39) and relieve hypoxia-caused damage via the downregulation of
miR-449a (40). However, the number of functional miRNAs involved in the development of isoflurane-induced cognitive dysfunction remains unknown.

miR-150 has been reported to exert protective effects in neuropathic pain by suppressing neuroinflammation (19). Moreover, aberrant expression of miR-150 has been observed in multiple sclerosis after treatment with curcumin (41). In the present study, it was found that the hippocampal expression of miR-150 was significantly decreased in rats exposed to isoflurane, and that this inhibitory effect was relieved by treatment with TMP, suggesting the potential role of miR-150 in the development of isoflurane-induced injury and in the biological function of TMP. Further experiments were performed to verified this conclusion, the results of which indicated that the overexpression of miR-150 in rats improved the impairment in cognitive function and inflammatory reaction induced by isoflurane exposure. Furthermore, the knockdown of miR-150 abrogated the protective effects of TMP on spatial learning and memory abilities, as well as neuroinflammation in rats. Collectively, these results suggested that miR-150 may be a mediator of the neuroprotective effects of TMP via neuroinflammation in rats exposed to isoflurane.

miRNAs are involved in the development of various human disease by regulating their target genes (42). In the present study, a number of candidate target genes were predicted using TargetScan for miR-150, and AKT3 was selected based on previous studies demonstrating its crucial role in neurological function $(43,44)$. AKT3 is a major isoform of the AKT family of serine/threonine protein kinases, which is predominant in the brain (45). The dysregulation of AKT3 has been widely reported to be involved in the regulation of learning and memory deficits in several diseases $(43,44)$. Additionally, AKT3 has been shown to be a target gene of miR-150 (19), which is one of the major molecules of the PI3K/AKT signaling pathway. In the present study, it was demonstrated that AKT3 was a direct target of miR-150, and the results indicated the negatively regulatory effect of miR-150 on the expression of AKT3 in rats exposed to isoflurane and administrated with TMP. It was also found that inhibited AKT3 expression induced by TMP could be rescued by the knockdown of miR-150, which suggested that miR-150 may mediate the neuroprotective 
effect of TMP in isoflurane-treated rats by targeting AKT3. $\mathrm{PI} 3 \mathrm{~K} / \mathrm{AKT}$ signaling is considered an important pathway in regulating neuroinflammation (46). Yang et al (47) revealed that TMP can ameliorate the injury induced by acute myocardial ischemia and attenuated the inflammatory response by regulating the PI3K/AKT signaling pathway. These previous studies combined with the current findings suggest that TMP may protect isoflurane-induced cognitive dysfunction via the miR-150/AKT signaling, and this conclusion warrants further investigation.

Alongside AKT3, other targets of miR-150 have previously been reported, such as glioma-associated oncogene homolog 1 (Gli1) (48) and hypoxia inducible factor-1 $\alpha$ (HIF-1 $\alpha$ ) (49). For instance, Peng et al (50) found that Gli1 was involved in the cerebral ischemia/reperfusion injury after isoflurane post-conditioning. Moreover, HIF-1 $\alpha$ has been reported to serve an important role in the blood-brain barrier disruption induced by isoflurane (51), and it could mediate the biological function of TMP in the improvement of tibial dyschodroplasia (52) and endothelial dysfunction (53). However, whether these aforementioned genes also participate the neuroprotective effects of TMP mediated by miR-150 remains unknown, and future studies should focus on the potential targets and signaling to further confirm the mechanisms underlying the functional role of TMP and miR-150 in isoflurane-induced cognitive dysfunction.

In conclusion, the present study provided evidence that TMP and miR-150 exert protective roles against the cognitive dysfunction induced by isoflurane in rats. TMP ameliorates the isoflurane-induced impairments in learning and memory via the attenuation of neuroinflammation by promoting the miR-150/AKT pathway. Moreover, miR-150 may serve as a potential therapeutic target for the alleviation of isoflurane-induced cognitive dysfunction.

\section{Acknowledgements}

Not applicable.

\section{Funding}

No funding was received.

\section{Availability of data and materials}

The datasets used and/or analyzed during the current study are available from the corresponding author on reasonable request.

\section{Authors' contributions}

HC and ZX made substantial contributions to conception and design, acquisition of data, analysis and interpretation of data. HC, ZX and CQ were involved in animal experiments, drafting the manuscript and revising it. All authors read and approved the final manuscript

\section{Ethics approval and consent to participate}

All the animal experiments were in accordance with the guidelines of the National Institutes of Health and Association for Assessment and Accreditation of Laboratory Animal Care, and were approved by the Animal Care and Use Committee of Dongying Hospital of Traditional Chinese Medicine Hospital.

\section{Patient consent for publication}

Not applicable.

\section{Competing interests}

The authors declare that they have no competing interests.

\section{References}

1. Chen L, Xie W, Xie W, Zhuang W, Jiang C and Liu N: Apigenin attenuates isoflurane-induced cognitive dysfunction via epigenetic regulation and neuroinflammation in aged rats. Arch Gerontol Geriatr 73: 29-36, 2017.

2. Sha HY, Zhao JB, Sha MX and Guo SM: Effects of vitamin B12 on postoperative cognitive dysfunction induced by isoflurane anesthesia in rats. Eur Rev Med Pharmacol Sci 21: 1959-1966, 2017.

3. Bartels K, Li YJ, Li YW, White WD, Laskowitz DT, Kertai MD, Stafford-Smith M, Podgoreanu MV, Newman MF and Mathew JP: Apolipoprotein epsilon 4 genotype is associated with less improvement in cognitive function five years after cardiac surgery: A retrospective cohort study. Can J Anaesth 62: 618-626, 2015

4. Song J, Chu S, Cui Y, Qian Y, Li X, Xu F, Shao X, Ma Z, Xia T and $\mathrm{Gu} \mathrm{X}$ : Circadian rhythm resynchronization improved isoflurane-induced cognitive dysfunction in aged mice. Exp Neurol 306: 45-54, 2018.

5. Guo M, Zhu X, Xu H, Li J, Yang S, Zuo Z and Lin D: Ulinastatin attenuates isoflurane-induced cognitive dysfunction in aged rats by inhibiting neuroinflammation and $\beta$-amyloid peptide expression in the brain. Neurol Res 41: 1-7, 2019.

6. Callaway JK, Wood C, Jenkins TA, Royse AG and Royse CF: Isoflurane in the presence or absence of surgery increases hippocampal cytokines associated with memory deficits and responses to brain injury in rats. Behav Brain Res 303: 44-52, 2016.

7. Wang W, Chen X, Zhang J, Zhao Y, Li S, Tan L, Gao J, Fang X and Luo A: Glycyrrhizin attenuates isoflurane-induced cognitive deficits in neonatal rats via its anti-inflammatory activity. Neuroscience 316: 328-336, 2016.

8. Shao Z, Wang L, Liu S and Wang X: Tetramethylpyrazine protects neurons from oxygen-glucose deprivation-induced death. Med Sci Monit 23: 5277-5282, 2017.

9. Liu Y, Li X, Jiang S and Ge Q: Tetramethylpyrazine protects against high glucose-induced vascular smooth muscle cell injury through inhibiting the phosphorylation of JNK, p38MAPK, and ERK. J Int Med Res 46: 3318-3326, 2018.

10. Shao Z, Wu P, Wang X, Jin M, Liu S, Ma X and Shi H: Tetramethylpyrazine protects against early brain injury and inhibits the PERK/Akt pathway in a rat model of subarachnoid hemorrhage. Neurochem Res 43: 1650-1659, 2018.

11. Michel HE, Tadros MG, Esmat A, Khalifa AE and Abdel-Tawab AM: Tetramethylpyrazine ameliorates rotenone-induced Parkinson's disease in rats: Involvement of its anti-inflammatory and anti-apoptotic actions. Mol Neurobiol 54: 4866-4878, 2017.

12. Xu SH, Yin MS, Liu B, Chen ML, He GW, Zhou PP, Cui YJ, Yang D and $\mathrm{Wu}$ YL: Tetramethylpyrazine-2'-O-sodium ferulate attenuates blood-brain barrier disruption and brain oedema after cerebral ischemia/reperfusion. Hum Exp Toxicol 36: 670-680, 2017.

13. Fu S, Wang J, Hao C, Dang H and Jiang S: Tetramethylpyrazine ameliorates depression by inhibiting TLR4-NLRP3 inflammasome signal pathway in mice. Psychopharmacology (Berl) 236: 2173-2185, 2019.

14. Wang Z, Wang Q, Wang C, Xu X and Yu H: Tetramethylpyrazine attenuates periorbital allodynia and neuroinflammation in a model of traumatic brain injury. J Inflamm (Lond) 14: 13, 2017.

15. Vienberg S, Geiger J, Madsen S and Dalgaard LT: MicroRNAs in metabolism. Acta Physiol (Oxf) 219: 346-361, 2017.

16. Rodrigues DC, Kim DS, Yang G, Zaslavsky K, Ha KC, Mok RS, Ross PJ, Zhao M, Piekna A, Wei W, et al: MECP2 is post-transcriptionally regulated during human neurodevelopment by combinatorial action of RNA-binding proteins and miRNAs. Cell Rep 17: 720-734, 2016. 
17. Gaudet AD, Fonken LK, Watkins LR, Nelson RJ and Popovich PG: MicroRNAs: Roles in regulating neuroinflammation. Neuroscientist 24: 221-245, 2018.

18. Ji LJ, Shi J, Lu JM and Huang QM: MiR-150 alleviates neuropathic pain via inhibiting toll-like receptor 5. J Cell Biochem 119: 1017-1026, 2018.

19. Cai W, Zhang Y, Liu Y, Liu H, Zhang Z and Su Z: Effects of miR-150 on neuropathic pain process via targeting AKT3. Biochem Biophys Res Commun 517: 532-537, 2019.

20. Wang J, Masika J, Zhou J, Wang J, Zhu M, Luo H, Hu X, Zhang L, Tang M, Gao L, et al: Traditional Chinese medicine baicalin suppresses mESCs proliferation through inhibition of miR-294 expression. Cell Physiol Biochem 35: 1868-1876, 2015.

21. Newcomer CE: The evolution and adoption of standards used by AAALAC. J Am Assoc Lab Anim Sci 51: 293-297, 2012.

22. Chang CY, Kao TK, Chen WY, Ou YC, Li JR, Liao SL, Raung SL and Chen CJ: Tetramethylpyrazine inhibits neutrophil activation following permanent cerebral ischemia in rats. Biochem Biophys Res Commun 463: 421-427, 2015.

23. Shi C, Yi D, Li Z, Zhou Y, Cao Y, Sun Y, Chui D and Guo X: Anti-RAGE antibody attenuates isoflurane-induced cognitive dysfunction in aged rats. Behav Brain Res 322: 167-176, 2017.

24. Livak KJ and Schmittgen TD: Analysis of relative gene expression data using real-time quantitative PCR and the 2(-Delta Delta C(T)) method. Methods 25: 402-408, 2001.

25. Shan L, Ma D, Zhang C, Xiong W and Zhang Y: miRNAs may regulate GABAergic transmission associated genes in aged rats with anesthetics-induced recognition and working memory dysfunction. Brain Res 1670: 191-200, 2017.

26. Li FS and Weng JK: Demystifying traditional herbal medicine with modern approach. Nat plants 3: 17109, 2017.

27. Zhang Y, Zhang Z, Wang H, Cai N, Zhou S, Zhao Y, Chen X, Zheng S, Si Q and Zhang W: Neuroprotective effect of ginsenoside Rg1 prevents cognitive impairment induced by isoflurane anesthesia in aged rats via antioxidant, anti-inflammatory and anti-apoptotic effects mediated by the PI3K/AKT/GSK-3beta pathway. Mol Med Rep 14: 2778-2784, 2016.

28. Chen L, Zhang B, Shan S and Zhao X: Neuroprotective effects of vitexin against isoflurane-induced neurotoxicity by targeting the TRPV1 and NR2B signaling pathways. Mol Med Rep 14: 5607-5613, 2016

29. Liang L, Ma Z, Dong M, Ma J, Jiang A and Sun X: Protective effects of salidroside against isoflurane-induced cognitive impairment in rats. Hum Exp Toxicol 36: 1295-1302, 2017.

30. Zhao S, Zhang Z, Yao Z, Shao J, Chen A, Zhang F and Zheng S: Tetramethylpyrazine attenuates sinusoidal angiogenesis via inhibition of hedgehog signaling in liver fibrosis. IUBMB life 69 : 115-127, 2017.

31. Zhou Y,JiZ, Yan W,ZhouZ,LiH and Xiao Y: Tetramethylpyrazine inhibits prostate cancer progression by downregulation of forkhead box M1. Oncol Rep 38: 837-842, 2017.

32. Zhang G, Zhang T, Li N, Wu L, Gu J, Li C, Zhao C, Liu W, Shan L, Yu P, et al: Tetramethylpyrazine nitrone activates the BDNF/Akt/CREB pathway to promote post-ischaemic neuroregeneration and recovery of neurological functions in rats. Br J Pharmacol 175: 517-531, 2018.

33. Zheng B, Lai R, Li J and Zuo Z: Critical role of P2X7 receptors in the neuroinflammation and cognitive dysfunction after surgery. Brain Behav Immun 61: 365-374, 2017.

34. Miao HH, Zhang Y, Ding GN, Hong FX, Dong P and Tian M: Ginsenoside Rb1 attenuates isoflurane/surgery-induced cognitive dysfunction via inhibiting neuroinflammation and oxidative stress. Biomed Environ Sci 30: 363-372, 2017.

35. Chen L, Liu T, Wang Q and Liu J: Anti-inflammatory effect of combined tetramethylpyrazine, resveratrol and curcumin in vivo. BMC Complement Altern Med 17: 233, 2017.

36. Sorrenti V, Giusti P and Zusso M: A model of systemic inflammation to study neuroinflammation. Methods Mol Biol 1727: $361-372,2018$
37. Liu J, Li M, Wang Y and Luo J: Curcumin sensitizes prostate cancer cells to radiation partly via epigenetic activation of miR-143 and miR-143 mediated autophagy inhibition. J Drug Target 25: 645-652, 2017

38. Fan L, Zhang H, Li X, Yang G, Ru J and Liu T: Emodin protects hyperglycemia-induced injury in PC-12cells by up-regulation of miR-9. Mol Cell Endocrinol 474: 194-200, 2018.

39. Fan $\mathrm{Y}$ and $\mathrm{Wu} \mathrm{Y}$ : Tetramethylpyrazine alleviates neural apoptosis in injured spinal cord via the downregulation of miR-214-3p. Biomed Pharmacother 94: 827-833, 2017.

40. Zhang X,Dong H,Liu Y,Han J,Tang S and Si J: Tetramethylpyrazine partially relieves hypoxia-caused damage of cardiomyocytes H9c2 by downregulation of miR-449a. J Cell Physiol: Feb 15, 2019 (Epub ahead of print). doi: 10.1002/jcp.28151.

41. Dolati S, Aghebati-Maleki L, Ahmadi M, Marofi F, Babaloo Z, Ayramloo H, Jafarisavari Z, Oskouei H, Afkham A, Younesi V, et al: Nanocurcumin restores aberrant miRNA expression profile in multiple sclerosis, randomized, double-blind, placebo-controlled trial. J Cell Physiol 233: 5222-5230, 2018

42. Chen X, Li Z, Xu D and Li S: LINC01121 induced intervertebral disc degeneration via modulating miR-150-5p/MMP16 axis. J Gene Med: May 21, 2020 (Epub ahead of print). doi: 10.1002/jgm.3231.

43. Zhang T, Shi Z, Wang Y, Wang L, Zhang B, Chen G, Wan Q and Chen L: Akt3 deletion in mice impairs spatial cognition and hippocampal CA1 long long-term potentiation through downregulation of mTOR. Acta Physiol (Oxf) 225: e13167, 2019.

44. Howell KR, Floyd K and Law AJ: PKBgamma/AKT3 loss-of-function causes learning and memory deficits and deregulation of AKT/mTORC2 signaling: Relevance for schizophrenia. PLoS One 12: e0175993, 2017.

45. DuBois JC, Ray AK, Gruber RC, Zhang Y, Aflakpui R, Macian-Juan F and Shafit-Zagardo B: Akt3-mediated protection against inflammatory demyelinating disease. Front Immunol 10: 1738,2019

46. Tang G, Yang H, Chen J, Shi M, Ge L, Ge X and Zhu G: Metformin ameliorates sepsis-induced brain injury by inhibiting apoptosis, oxidative stress and neuroinflammation via the PI3K/Akt signaling pathway. Oncotarget 8: 97977-97989, 2017.

47. Yang Q, Huang DD, Li DG, Chen B, Zhang LM, Yuan CL and Huang $\mathrm{HH}$ : Tetramethylpyrazine exerts a protective effect against injury from acute myocardial ischemia by regulating the PI3K/Akt/GSK-3beta signaling pathway. Cell Mol Biol Lett 24: 17, 2019.

48. Fan H, Liu X, Zheng WW, Zhuang ZH and Wang CD: MiR-150 alleviates EMT and cell invasion of colorectal cancer through targeting Gli1. Eur Rev Med Pharmacol Sci 21: 4853-4859, 2017.

49. Li Y, Su J, Li F, Chen X and Zhang G: MiR-150 regulates human keratinocyte proliferation in hypoxic conditions through targeting HIF-1alpha and VEGFA: Implications for psoriasis treatment. PLoS One 12: e0175459, 2017.

50. Peng L, Yang C, Yin J, Ge M, Wang S, Zhang G, Zhang Q, Xu F, Dai Z, Xie L, et al: TGF- $\beta 2$ induces Gli1 in a Smad3-dependent manner against cerebral ischemia/reperfusion injury after isoflurane post-conditioning in rats. Front Neurosci 13: 636, 2019.

51. Cao Y, Li Z, Li H, Ni C, Li L, Yang N, Shi C, Zhong Y, Cui D and Guo $\mathrm{X}$ : Hypoxia-inducible factor-1alpha is involved in isoflurane-induced blood-brain barrier disruption in aged rats model of POCD. Behav Brain Res 339: 39-46, 2018.

52. Mehmood K, Zhang H, Li K, Wang L, Rehman MU, Nabi F, Iqbal MK, Luo H, Shahzad M and Li J: Effect of tetramethylpyrazine on tibial dyschondroplasia incidence, tibial angiogenesis, performance and characteristics via HIF-1alpha/VEGF signaling pathway in chickens. Sci Rep 8: 2495, 2018.

53. Yang C, Xu Y, Zhou H, Yang L, Yu S, Gao Y, Huang Y, Lu L and Liang X: Tetramethylpyrazine protects $\mathrm{CoCl} 2$-induced apoptosis in human umbilical vein endothelial cells by regulating the PHD2/HIF/1 $\alpha$-VEGF pathway. Mol Med Rep 13: 1287-1296, 2016. 